\title{
Evaluation of the anterior chamber angle in pseudoexfoliation syndrome
}

\author{
Małgorzata Iwanejko ${ }^{1, A-C, F}$, Anna Turno-Kręcicka ${ }^{1, D-F}$, Martyna Tomczyk-Socha ${ }^{1,0, E}$, \\ Kamil Kaczorowski1 ${ }^{1, \mathrm{~F}}$, Andrzej Grzybowski ${ }^{2,3}$,F, Marta Misiuk-Hojło ${ }^{1, F}$ \\ ${ }^{1}$ Department and Clinic of Ophthalmology, Wroclaw Medical University, Poland \\ ${ }^{2}$ Department of Ophthalmology, Poznan City Hospital, Poznań, Poland \\ ${ }^{3}$ Deptartment of Ophthalmology, University of Warmia and Mazury, Olsztyn, Poland \\ A - research concept and design; $\mathrm{B}$ - collection and/or assembly of data; $\mathrm{C}$ - data analysis and interpretation; \\ $D$ - writing the article; $E$ - critical revision of the article; $F$ - final approval of article
}

Address for correspondence

Kamil Kaczorowski

E-mail: drkamilkaczorowski@gmail.com

Funding sources

None declared

Conflict of interest

None declared

Received on August 17, 2015

Revised on September 29, 2015

Accepted on July 04,2016

\section{Abstract}

Background. Pseudoexfoliation syndrome (PEX) is the most frequently identifiable cause of secondary open-angle glaucoma, known as pseudoexfoliation glaucoma. The exact pathophysiology and etiology of PEX and associated glaucoma remains obscure.

Objectives. The purpose of this study was to determine the differences in the morphology of the anterior chamber angle in people with pseudoexfoliation syndrome and pseudoexfoliation glaucoma compared to a control group. We also evaluated the correlation between intraocular pressure (IOP) and pigmentation of the angle with the amount of exfoliated material in the anterior segment.

Material and methods. The study group was composed of 155 eyes from 103 patients aged between 43 and 86 years. Each patient underwent a complete ophthalmological examination.

Results. Some difference was found in intraocular pressure between the PEX group and the control group and between the pseudoexfoliation glaucoma group and the control group, but no significant difference was found between the 2 study groups. There was a significant difference in the incidence of some degree of pigmentation in the anterior chamber angle and no difference in the widths of the angle between each group. A significant positive relationship was observed between intraocular pressure and the degree of pigmentation of the anterior chamber angle in both the PEX group and the pseudoexfoliation glaucoma group.

Conclusions. The results of this study indicate that the amount of pigmentation and exfoliation material in the anterior segment significantly correlates with the level of IOP and possibly with the degree of trabecular dysfunction. It seems that for clear identification of PEX and pseudoexfoliation glaucoma factors, clinical assessment appears to be insufficient.

Key words: pseudoexfoliation syndrome, PEX, pseudoexfoliation glaucoma, PEXG, morphology of the ocular angle

DOI

10.17219/acem/64023

\section{Copyright}

Copyright by Author(s)

This is an article distributed under the terms of the

Creative Commons Attribution Non-Commercial License

(http://creativecommons.org/licenses/by-nc-nd/4.0/) 
Pseudoexfoliation syndrome (PEX) is an age-related systemic disease which is characterized by excessive production and accumulation of amyloid-like protein material caused by the degeneration of elastic fibers of the connective tissue. Exfoliation material (EXM) is produced by pathological cells of the connective tissue. It accumulates in the anterior segment of the eye and in other tissues outside the eyeball, for example in blood vessels, the heart, lungs or kidneys. PEX occurs worldwide, but its geographical distribution is differentiated. It is estimated that approximately 70 million people worldwide suffer from PEX. ${ }^{1,2}$

PEX is the most frequently identifiable cause of secondary open-angle glaucoma, known as pseudoexfoliation glaucoma (PEXG). ${ }^{3,9}$ However, the conversion agent from PEX to PEXG still remains unknown-glaucoma is identified in about $30-40 \%$ of PEX cases. ${ }^{3,10,11}$ The prognosis of glaucoma associated with PEX is generally worse in comparison with other types of glaucoma due to its more dynamic course. It may be characterized by high intraocular pressure, which can imitate an attack of acute angleclosure glaucoma, although the angle is usually open. ${ }^{3,10}$ Treatment of PEXG is considered to be difficult and often leads to failure due to an unsatisfactory response to treatment or a large number of cases with higher than expected intraocular pressure (IOP) in glaucoma and its diurnal variation. ${ }^{11,12}$

Despite intensive research and medical progress, the exact pathophysiology and etiology of PEX and the glaucoma which is associated with this syndrome still remain obscure. $^{3-5}$ The development of PEXG likely has a multifactorial substrate. The pathological formation of elastosis leads to a disorder in the structure of Schlemm's canal, the trabecular meshwork and the cribriform plate of the ethmoid bone. Moreover, vascular changes in the described rebuilding cause insufficient blood circulation in the front part of the optic nerve. Additionally, mechanical obstruction by the EXMs and pigment grains released from the iris pigment epithelium block the drainage of the aqueous humor during mydriasis. ${ }^{13}$

Due to the fact that PEX is an important indicator of the possibility of glaucomatous optic neuropathy, and that no factor leading to the conversion from PEX to PEXG has been identified so far, finding its symptoms requires regular examination. ${ }^{3,13}$ Discovering symptoms which occur before the conversion takes place could be very helpful in deciding whether to examine a patient more frequently or to implement glaucoma treatment earlier.

The purpose of this study was to determine the differences in the morphology of the anterior chamber angle, examined and described through gonioscopy, in people with pseudoexfoliation syndrome and pseudoexfoliation glaucoma compared to a control group. We also evaluated the correlation between the level of intraocular pressure and the pigmentation of the angle with the amount of EXM in the anterior segment.
Another aspect was to determine the differences in the angle obtained by an optical coherence tomography (OCT) test of the anterior segment of the eye after it adapts to darkness.

\section{Material and methods}

This prospective, controlled study was approved by the Regional Ethical Review Board and was conducted in accordance with the principles set forth in the Guidelines for Good Clinical Practice and the Declaration of Helsinki (and its amendments). ${ }^{14}$

The study group was composed of 155 eyes from 103 patients (77 women and 26 men) aged between 43 and 86 years (the average age was $68.83 \pm 9.68$ ). The patients were consecutively selected and assigned to one of the 3 groups:

1) a control group of healthy patients $(C): 35$ people, 63 eyes;

2) a group of patients with pseudoexfoliation syndrome (PEX): 38 people, 50 eyes; or

3) a group of patients with pseudoexfoliation syndrome and glaucoma (PEXG): 30 people, 42 eyes.

The groups did not differ significantly in terms of age and gender make-up, but women outnumbered men in all groups. The surface of the optic disc and visual acuity showed no statistically significant differences between the groups (Table 1).

Each patient underwent a complete ophthalmological examination comprising an interview, anterior segment slit lamp biomicroscopy before and after dilation of the pupil and measurement of intraocular pressure (IOP) by Goldmann applanation tonometry - the average of 3 measurements was used for further calculations. Additionally, central corneal pachymetry (Opticon 2000) and optical coherence tomography (OCT) (Visante 1000) were performed. In gonioscopy (Zeiss gonioscopy), the angle was divided into 4 quadrants and classified according to the Spaeth Grading System. The amount of pigment was evaluated according to the following scale:

- 0 - none,

- I - some browning,

- II - firm brown pigmentation,

- III - dark brown or black pigmentation, presence or absence of Sampaolesi line.

The amount of exfoliation material was reported according to the following scale:

- 0 - none,

- I - exfoliation confined to the periphery of the lens and not seen unless the pupil is dilated,

- II - exfoliated material on the edge of the iris or on the surface of the lens capsule or both,

- III - grade II and exfoliated material in the angle.

In order to compare the width of the anterior chamber angle, measured through the use of gonioscopy and OCT, the average value of angular widths on 3 . and 9 . h was used. 
Table 1. Comparative analysis of the study groups: control (C), patients with pseudoexfoliation syndrome (PEX), patients with pseudoexfoliation syndrome and glaucoma (PEXG)

\begin{tabular}{|l|c|c|c|}
\hline \multirow{2}{*}{ 34. } & \multicolumn{3}{|c|}{ Study groups } \\
\cline { 2 - 4 } & C & PEX & P-value \\
\hline Number of eyes & 63 & 50 & 42 \\
\hline Gender (woman /man) & $28(80 \%) / 7(20 \%)$ & $27(28.9 \%) / 11(71.1 \%)$ & $22(26.7 \%) / 8(73.3 \%)$ \\
\hline Visual acuity & $0.98 \pm 0.04$ & $0.97 \pm 0.05$ & $0.92 \pm 0.12$ \\
\hline Surface of the optic disc & $2.29 \pm 0.37 \mathrm{~mm}^{2}$ & $2.39 \pm 0.44 \mathrm{~mm}^{2}$ & $2.43 \pm 0.45 \mathrm{~mm}^{2}$ \\
\hline
\end{tabular}

Table 2. Incidence of Sampaolesi line in C, PEX and PEXG groups

\begin{tabular}{|c|c|c|c|}
\hline \multicolumn{1}{|c|}{ Group } & C & PEX & PEXG \\
\hline- & 63 & 35 & 25 \\
\hline$\%$ & $100.00 \%$ & $65.00 \%$ & $60.52 \%$ \\
\hline+ & 0 & 15 & 17 \\
\hline$\%$ & $0.00 \%$ & $35.00 \%$ & $39.48 \%$ \\
\hline
\end{tabular}

Table 3. Occurrence of the specific attachment of the iris

\begin{tabular}{|l|c|c|c|}
\multirow{2}{*}{ Type } & \multicolumn{3}{|c|}{ Groups } \\
\cline { 2 - 4 } D & C & PEX & PEXG \\
\hline$\%$ & 43 & 28 & 28 \\
\hline C & 68.25 & 56.00 & 66.67 \\
\hline$\%$ & 17 & 12 & 8 \\
\hline B & 26.98 & 24.00 & 19.05 \\
\hline$\%$ & 3 & 10 & 6 \\
\hline A & 4.76 & 18.00 & 14.29 \\
\hline \% & 0.00 & 2.00 & 0 \\
\hline
\end{tabular}

\section{Statistical analysis}

The parameters (IOP, degree of pigmentation, AOD 500 and 750, TISA 500 and 750, widths of the angle) were compared using a non-parametric Kruskal-Wallis test and a multiple comparison Kruskal-Wallis test. The qualitative data (incidence of Sampaolesi line, occurrence of pseudoexfoliation material, occurrence of specific attachments of the iris and iris configuration) were compared using a $\chi^{2}$ test. Specific attachment of the iris and iris configuration were recorded as percentages. A Spearman's rank correlation test was used to examine correlation between variables. The re- sults were considered statistically significant at a p-value of less than 0.05. All calculations were carried out using STATISTICA v. 9.0 software. (StatSoft Inc., Tulsa, USA).

\section{Results}

A statistically significant difference $(\mathrm{p}<0.0001)$ was found in IOP between the PEX and C groups, and between the PEXG and C groups, while there was no statistically significant difference between the PEX and PEXG groups ( $\mathrm{p}=0.1778)$ (Fig. 1$)$.

There was a difference in the incidence of some degree of pigmentation of the anterior chamber angle between the PEX and C groups ( $<<0.0001)$, the PEXG and $C$ groups ( $p<0.0001)$, and between the PEX and PEXG groups ( $p=0.0012)$ (Fig. 2). A difference was found in the incidence of Sampaolesi line between the groups $(\mathrm{p}<0.0001)$ (Table 2).

A difference was noted in the occurrence of pseudoexfoliation material between the tested groups ( $\mathrm{p}<0.0001)$. The control group showed an absence of the material, while a similar occurrence was observed in PEX and PEXG groups ( $\mathrm{p}=1$ ) (Fig. 3 ).

There was no difference in the frequency of specific attachment of the iris between the PEX and PEXG groups ( $\mathrm{p}=0.2343$ ) (Table 3).

Iris configuration differed significantly among the tested groups $(\mathrm{p}=0.0094)$ : configuration $S$ was the most frequent configuration in the PEX and PEXG groups $(14 \%$ and $11 \%$, respectively), but the rarest in the control group (approx. 5\%) (Table 4).

A significant, strong positive relationship was observed between IOP and the degree of pigmentation of the anterior chamber angle in the PEX group $(\mathrm{p}<0.0001$, $\mathrm{R}=0.729$ ) (Fig. 4a), as well as in the PEXG group ( $\mathrm{p}<0.0001, \mathrm{R}=0.598$ ) (Fig. 4b).

There was a significant, strong positive relationship between IOP and the amount of pseudoexfoliation material on the front surface of the lens capsule and in the anterior chamber angle in the PEX group $(\mathrm{p}<0.0001, \mathrm{R}=0.7011)$ (Fig. 5a) and a significant positive correlation in the PEXG group ( $\mathrm{p}<0.0029, \mathrm{R}=0.4479$ ) (Fig. $5 \mathrm{~b}$ ). 
Fig. 1. Distribution of intraocular pressure found in C, PEX and PEXG groups

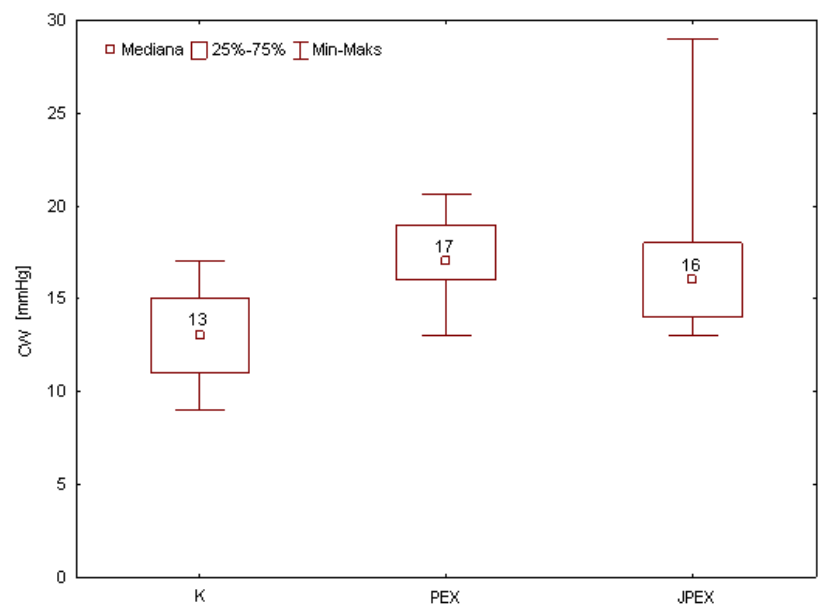

Fig. 2. Distribution of the degree of pigmentation of the angle in $C$, PEX and PEXG groups

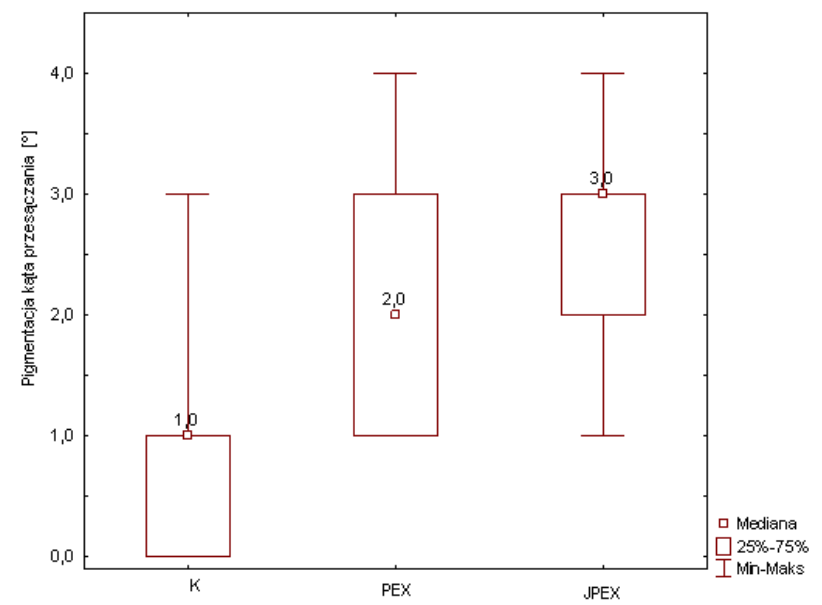

Fig. 3. Comparative summary of the degree of exfoliation in the anterior segment in C, PEX and PEXG groups
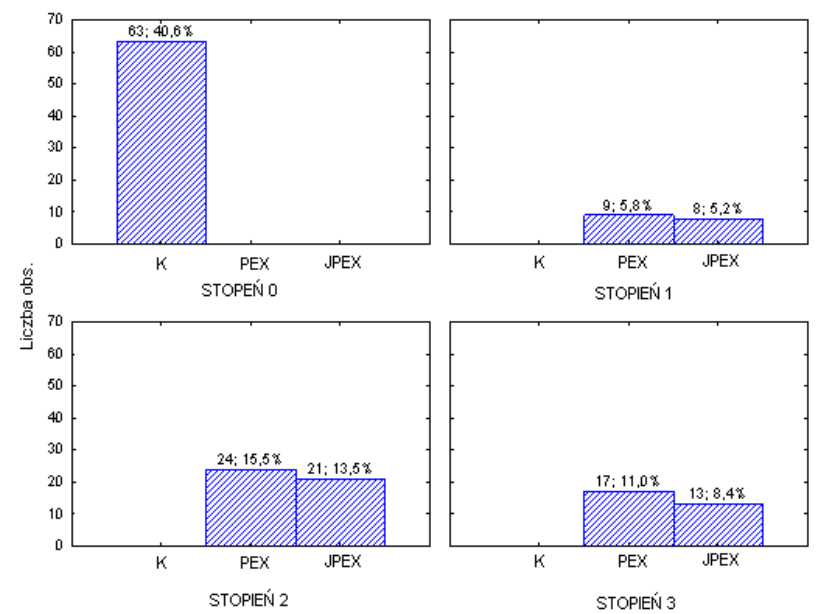

Fig. 4a. Intraocular pressure (IOP) and degree of pigmentation in PEX group

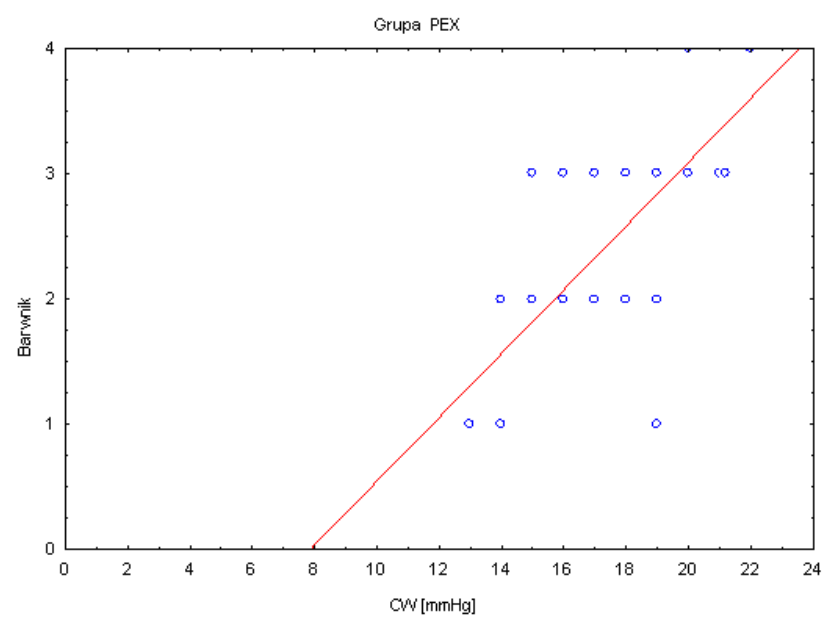

Fig. 4b. Intraocular pressure (IOP) and degree of pigmentation in PEXG group

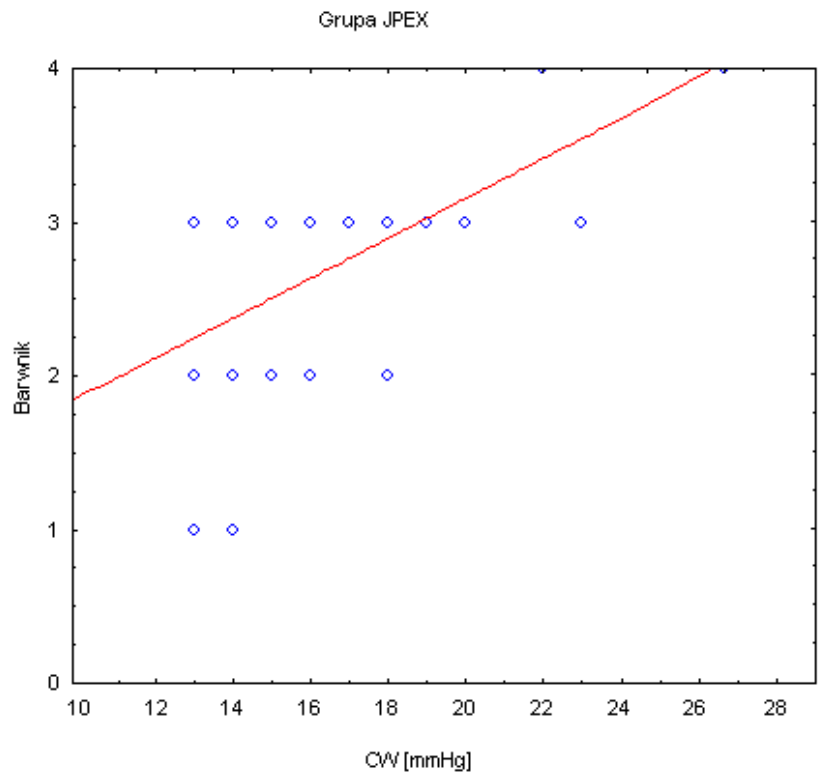

There was no statistically significant difference in the values of AOD at $500 \mu \mathrm{m}$ and $750 \mu \mathrm{m}$ and TISA at $500 \mu \mathrm{m}$ and at $750 \mu \mathrm{m}$, measured from the temple and from the nose by OCT Visante, between the C, PEX and PEXG groups $(\mathrm{p}=1)$.

There was no statistically significant difference between the widths of the anterior chamber angle in the $\mathrm{C}, \mathrm{PEX}$ and PEXG groups as measured by OCT Visante $(\mathrm{p}=0.8081)$ nor by gonioscopy $(\mathrm{p}=0.8469)$.

\section{Discussion}

In our study, no statistical difference was found in the amount of EXM between the PEX and PEXG groups, which may suggest that a higher amount of EXM does 
Fig. 5a. IOP and amount of pseudoexfoliation material on the front surface of the lens capsule and in the angle in the PEX group

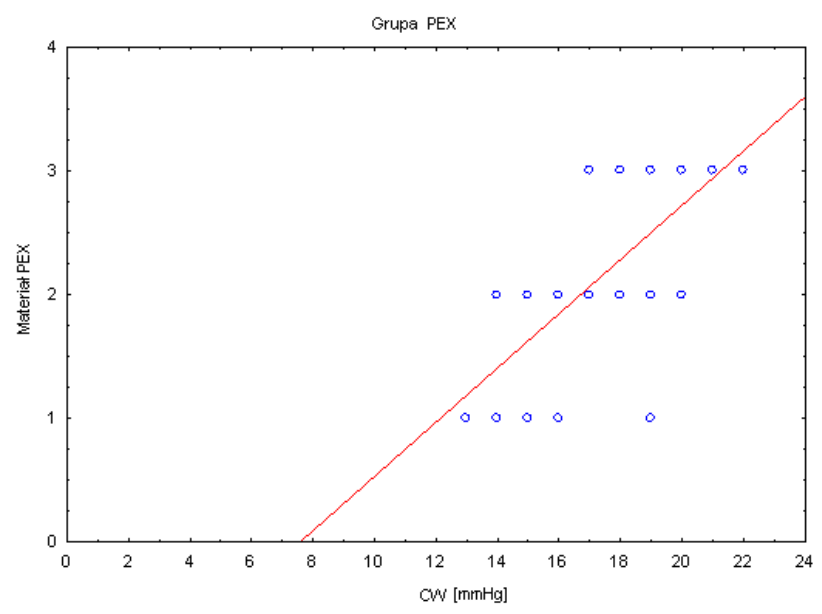

Fig. 5b. IOP and amount of pseudoexfoliation material on the front surface of the lens capsule and in the angle in the PEXG group

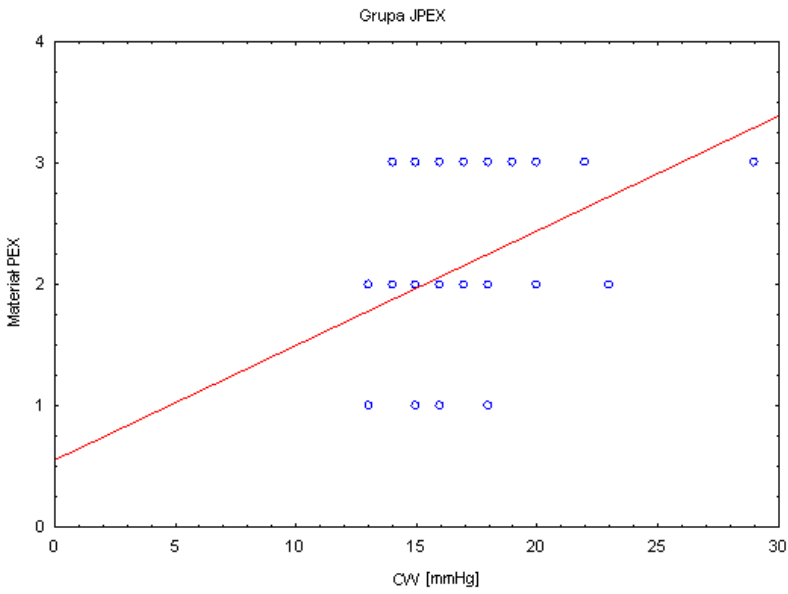

not necessarily determine glaucoma conversion. However, a positive correlation between the amount of EXM and the level of IOP was found in both the PEX and PEXG groups. A difference in the frequency of some degree of pigmentation was observed between groups $C$ and PEX, between $C$ and PEXG and between PEX and PEXG. The differences between groups were statistically significant, which suggests the importance of this parameter in distinguishing different groups. A statistically significant correlation also occurred between the amount of pigment and IOP in the PEX and PEXG groups.

One of the main risk factors for the development of both primary angle-closure glaucoma (POAG) and PEXG is an increased level of IOP.,16 There were statistically significant differences $(p<0.0001)$ between the PEX and $C$ groups and between PEXG and C. IOP was found to be significantly higher in the groups with PEX and PEXG compared to the control group, whereas there were no statistically significant differences between the study groups ( $\mathrm{p}=0.1778$ ). This finding could suggest quite a good response to treatment in patients from the
PEXG group; however, the interview shows that $57.2 \%$ of patients with PEXG (24 patients) experienced increases of IOP above $21 \mathrm{~mm} \mathrm{Hg}$ despite the treatment.

Four patients (11.4\%) had IOP higher than $22 \mathrm{~mm} \mathrm{Hg}$ despite several modifications to the treatment. Many researchers have also observed a statistically significantly higher IOP in patients with PEX compared to the control group. ${ }^{2,3,15,17,18}$ Klemetti et al. pointed out that the initial average IOP in eyes with PEX is important in the conversion from PEX to PEXG. They noticed that the eyes with PEX which developed ocular hypertension or glaucoma later in the course of disease had had a significantly higher IOP $(\mathrm{p}<0.001)$ compared to the eyes in which there was no development of glaucoma. ${ }^{19}$

Grodum et al. suggested that conversion to glaucoma was twice as fast in patients with PEX and ocular hypertension compared with healthy people, properly selected by gender and age. They also argued that an elevated level of IOP during diagnosis seemed to be one of the strongest risk factors for conversion from PEX to PEXG. ${ }^{10}$ Konstas et al. demonstrated the benefits of lowering IOP and suggested that maintaining it below $17 \mathrm{~mm} \mathrm{Hg}$ in patients with PEX reduced the probability of conversion and could have slowed the progression of glaucomatous optic neuropathy that had already occurred. ${ }^{19}$

A large diurnal fluctuation in IOP seems to be one of the most important risk factors of PEXG. ${ }^{18,21,22}$ Nenciu et al. noted that it was much higher in patients with PEX than the physiological variability in healthy individuals. ${ }^{21}$

Another problem related to PEX is the possibility of an increased level of IOP during diagnostic pupil dilation, due to the scattering of grains of pigment and the increase associated with the change of body position. ${ }^{23,24}$ These aspects, however, were not examined in this study. The influence of elevated IOP in the case of PEXG may be higher than in the case of POAG because changes in elastosis formation in the cribriform plate of the ethmoid bone and in orbital vessels may cause increased sensitivity to optic nerve damage in comparison with the control group at the same age.,13 The increase of IOP and its diurnal variation appears to be an important prognostic factor occurring before the conversion from PEX to PEXG.

The correlation between increased pigmentation and the presence of PEX and PEXG has also been described by others. ${ }^{25,26}$ Puska et al. have found that eyes with PEXG show more pigmentation in the angle than eyes with glaucoma but without PEX. In addition, they noted that pigmentation was stronger in eyes with PEXG than in eyes with PEX, although the amount of EXM was similar. Moreover, they showed a positive correlation between IOP and the degree of pigmentation as well as between IOP and the amount of EXM. Because the statistical significance for the latter was smaller, it was concluded that the main risk factor for glaucoma seems to be the degree of pigmentation of the angle glaucoma. ${ }^{24}$ 
Moreno-Montañés et al. observed a statistically significant relationship between the pigmentation of the anterior chamber angle and IOP, while they found no relationship between the amount of EXM and the level of IOP. ${ }^{26}$ On the other hand, it is not known whether the degree of angle pigmentation and the amount of EXM correlates with the severity of glaucoma. ${ }^{27}$ Shuba et al. presented a positive correlation between pigmentation of the angle and IOP ( $p=0.047)$ and no correlation with the severity of glaucoma ( $p>0.13)$. There was no significant correlation between the amount of EXM and the level of IOP, nor a correlation with glaucoma severity indicators $(\mathrm{p}>0.04) .{ }^{28}$ Differences in these correlations may result from the classification system used in this study to determine the amount of EXM. The authors evaluated the amount of material on the front surface of the lens, which may not reflect the actual amount of material that blocks the drainage of the anterior chamber angle. ${ }^{3,29}$ Despite the absence of histological examination (clinical only), our results suggest that the amount of pigment and EXM in the angle can be a more important factor in the growth rate of IOP than the presence of PEX material in the anterior lens capsule only. From a clinical point of view, this highlights the importance of gonioscopy in the eyes of patients with PEX.

A Sampaolesi line was observed in both study groups, PEX and PEXG, and the difference between the groups was not statistically significant $(\mathrm{p}>0.05)$. This finding confirms that the Sampaolesi line is associated with the presence of pseudoexfoliation syndrome, but does not determine the presence of glaucoma. ${ }^{27}$

PEXG is classified as secondary open-angle glaucoma. However, a higher incidence of narrow- or closed-angle glaucoma in patients with PEX was reported: $18 \%$ of patients had narrow-angle, 14\% had closed-angle, and $37.83 \%$ of patients had angle closure. ${ }^{30,31}$ Due to the differences in the width of the angle, we attempted to verify the incidence of narrow angles using the OCT apparatus of the anterior segment because, despite the obvious advantages of gonioscopy in the estimation of the width of the angle, it is quite a subjective examination and the result may be affected, for example, by light intensity, by the force which gonioscopy exerts on the cornea or by the experience of the examiner. ${ }^{32}$ There was no statistical difference between the widths of the angle in the 2 groups measured by OCT $(\mathrm{p}>0.1)$. In all groups, the angle was open. Although several characteristic features could predispose a patient to angle-closure glaucoma in PEX (instability of the lens zonules, periodic forward movement of the lens or a greater tendency to form rear adhesions), the higher incidence of narrow angles in PEX patients examined by gonioscopy and OCT did not confirm this assumption. ${ }^{29}$ Analyzing the parameters of the angle in OCT, there was no statistical difference between the parameters AOD500, AOD750 TISA750 and TISA500. Even though the diameter of the pupil in the dark in the
PEX and PEXG groups was similar, it was significantly lower than in the control group $(p=0.012)$. Zheng et al. also found no statistical difference in ACA, AOD500, TIA500 and TISA500 in patients with PEX compared to healthy individuals examined in a dark room. ${ }^{33}$ Impairment of mydriasis in the PEX and PEXG groups was apparent, probably associated with an increased firmness of the iris (due to the accumulation of PEX material in the stroma), but the parameters of the angle were not statistically significant. It should be remembered, however, that the image of the angle is affected by the anatomical structures of the anterior segment of the eye. Assessment of the angle was insufficient to fully interpret the results; therefore, further examination of the anterior segment through OCT is needed for patients with PEX.

\section{Conclusions}

The results of this study indicate that the amount of pigment and exfoliation material in the anterior segment significantly correlates with the level of IOP and possibly with the degree of trabecular dysfunction. This highlights the importance of performing gonioscopy in patients with PEX. We did not find any differences in the width of the angle or in the parameters measured by OCT among the groups.

It seems that in order to make a clear identification of the factors which would lead to the conversion from PEX to PEXG, clinical assessment, although extremely important, appears to be insufficient.

\section{References}

1. Wang W, He M, Zhou M, et al. Ocular pseudoexfoliation syndrome and vascular disease: A systematic review and meta-analysis. PLoS One. 2014;9(3):e92767.

2. Angelilli A, Ritch R. Directed therapy for exfoliation syndrome. Open Ophthalmol J. 2009;3:70-74.

3. Schlötzer-Schrehardt U. Genetics and genomics of pseudoexfoliation syndrome/glaucoma. Middle East Afr J Ophthalmol. 2011;18(1):30-36.

4. Anastasopoulos E, Topouzis F, Wilson MR, et al. Characteristics of pseudoexfoliation in the Thessaloniki eye study. J Glaucoma. 2011;20(3):160-166.

5. Arnarsson A, Damji KF, Sverrisson T, et al. Pseudoexfoliation in the Reykjavik eye study: Prevalence and related ophthalmological variables. Acta Ophthalmol Scand. 2007;85(8):822-827.

6. Anastasopoulos E, Coleman AL, Wilson MR, et al. Association of LOXL1 polymorphisms with pseudoexfoliation, glaucoma, intraocular pressure, and systemic diseases in a Greek population. The Thessaloniki eye study. Invest Ophthalmol Vis Sci. 2014;55(7):4238-4243.

7. Topouzis F, Harris A, Wilson MR, et al. Increased likelihood of glaucoma at the same screening intraocular pressure in subjects with pseudoexfoliation: The Thessaloniki eye study. Am J Ophthalmol. 2009;148(4):606-613.

8. Shazly TA, Farrag AN, Kamel A, et al. Prevalence of pseudoexfoliation syndrome and pseudoexfoliation glaucoma in Upper Egypt. BMC Ophthalmol. 2011;11:18.

9. Aptel F, Denis P. Optical coherence tomography quantitative analysis of iris volume changes after pharmacologic mydriasis. Ophthalmology. 2010;117(1):3-10.

10. Grødum K, Heijl A, Bengtsson B. Risk of glaucoma in ocular hypertension with and without pseudoexfoliation. Ophthalmology. 2005;112(3):386-390. 
11. Koz OG, Turkcu MF, Yarangumeli A, et al. Normotensive glaucoma and risk factors in normotensive eyes with pseudoexfoliation syndrome. J Glaucoma. 2009;18(9):684-688.

12. Schlötzer-Schrehardt U, Naumann GOH. Ocular and systemic pseudoexfoliation syndrome. Am J Ophthalmol. 2006;141(5):921-937.

13. Schlötzer-Schrehardt U. New pathogenetic insights into pseudoex foliation syndrome/glaucoma. Therapeutically relevant? Ophthalmologe. 2012;109(10):944-951

14. World Medical Association: World Medical Association Declaration of Helsinki: Ethical principles for medical research involving human subjects. JAMA. 2000;284:3043-3045.

15. Madden JG, Crowley MJ. Factors in the exfoliation syndrome. Br J Ophthalmol. 1982;66(7):432-437.

16. Koucheki $B$, Hashemi H. Selective laser trabeculoplasty in the treatment of open-angle glaucoma. J Glaucoma. 2012;21(1):65-70.

17. Yüksel $N$, Altintaş $O$, Celik $M$, et al. Analysis of retinal nerve fiber layer thickness in patients with pseudoexfoliation syndrome using optical coherence tomography. Ophthalmologica. 2007;221(5):299-304.

18. Altintaş O, Yüksel N, Karabaş VL, et al. Diurnal intraocular pressure variation in pseudoexfoliation syndrome. Eur J Ophthalmol. 2004;14(6):495-500.

19. Klemetti A. Intraocular pressure in exfoliation syndrome. Acta Ophthalmol Suppl. 1988;184:54-58.

20. Jeng SM, Karger RA, Hodge DO, et al. The risk of glaucoma in pseu doexfoliation syndrome. J Glaucoma. 2007;16(1):117-121.

21. Nenciu A, Stefan C, Melinte D, et al. IOP diurnal fluctuations in patients presenting pseudoexfoliative syndrome. Oftalmologia. 2006;50(2):121-125.

22. Gumus K, Bozkurt B, Sonmez B, et al. Diurnal variation of intraocular pressure and its correlation with retinal nerve fiber analysis in Turkish patients with exfoliation syndrome. Graefes Arch Clin Exp Ophthalmol. 2006;244(2):170-176.
23. Puska P, Harju M. Optic nerve head topography in nonglaucomatous, normotensive patients with unilateral exfoliation syndrome. Graefes Arch Clin Exp Ophthalmol. 2009; 247(8):1111-1117.

24. Puska P. Unilateral exfoliation syndrome: conversion to bilateral exfoliation and to glaucoma: A prospective 10-year follow-up study. J Glaucoma. 2002;11(6):517-524.

25. Ekström C, Alm A. Pseudoexfoliation as a risk factor for prevalent open-angle glaucoma. Acta Ophthalmol. 2008;86(7):741-746.

26. Moreno-Montañés J, Quinteiro Alonso A, Alvarez Serna A, et al. Exfoliation syndrome: Clinical study of the irido-corneal angle. J Fr Ophtalmol. 1990;13(4):183-188.

27. Cobb CJ, Blanco GC, Spaeth GL. Exfoliation syndrome angle characteristics: A lack of correlation with amount of disc damage. Br J Ophthalmol. 2004;88(8):1002-1003.

28. Shuba L, Nicolela MT, Rafuse PE. Correlation of capsular pseudoexfoliation material and iridocorneal angle pigment with the severity of pseudoexfoliation glaucoma. J Glaucoma. 2007;16(1):94-97.

29. Ritch R, Schlötzer-Schrehardt U, Konstas AGP. Why is glaucoma associated with exfoliation syndrome? Prog Retin Eye Res. 2003;22(3):253-275.

30. Bagniewska-Iwanier M, Czajkowski J. Różnokierunkowe spojrzenie na zespół pseudoeksfoliacji (PEX). Okulistyka. 2005;(1):31-34.

31. Wishart PK, Spaeth GL, Poryzees EM. Anterior chamber angle in the exfoliation syndrome. Br J Ophthalmol. 1985;69(2):103-107.

32. Müller M, Dahmen G, Pörksen $E$, et al. Anterior chamber angle measurement with optical coherence tomography: Intraobserver and interobserver variability. J Cataract Refract Surg. 2006;32(11): 1803-1808.

33. Zheng X, Sakai H, Goto T, et al. Anterior segment optical coherence tomography analysis of clinically unilateral pseudoexfoliation syndrome: Evidence of bilateral involvement and morphologic factors related to asymmetry. Invest Ophthalmo/ Vis Sci. 2011;52(8):5679-5984. 